BMJ Open

Diabetes

Research

\& Care

\section{Large-scale survey of rates of achieving targets for blood glucose, blood pressure, and lipids and prevalence of complications in type 2 diabetes (JDDM 40)}

To cite: Yokoyama $\mathrm{H}$, Oishi M, Takamura $\mathrm{H}$, et al. Large-scale survey of rates of achieving targets for blood glucose, blood pressure, and lipids and prevalence of complications in type 2 diabetes (JDDM 40). BMJ Open Diabetes Research and Care 2016;4:e000294. doi:10.1136/bmjdrc-2016000294

- Additional material is available. To view please visit the journal (http://dx.doi.org/ 10.1136/bmjdrc-2016000294)

Received 6 July 2016 Revised 10 August 2016 Accepted 12 September 2016

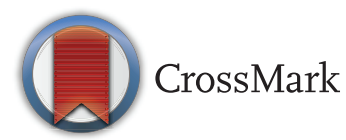

For numbered affiliations see end of article.

Correspondence to Dr Hiroki Yokoyama; dryokoyama@yokoyamanaika. com

\section{ABSTRACT}

Objective: The fact that population with type 2 diabetes mellitus and bodyweight of patients are increasing but diabetes care is improving makes it important to explore the up-to-date rates of achieving treatment targets and prevalence of complications. We investigated the prevalence of microvascular/ macrovascular complications and rates of achieving treatment targets through a large-scale multicenterbased cohort.

Research design and methods: A cross-sectional nationwide survey was performed on 9956 subjects with type 2 diabetes mellitus who consecutively attended primary care clinics. The prevalence of nephropathy, retinopathy, neuropathy, and macrovascular complications and rates of achieving targets of glycated hemoglobin $(\mathrm{HbA} 1 \mathrm{c})<7.0 \%$, blood pressure $<130 / 80 \mathrm{~mm} \mathrm{Hg}$, and lipids of low-density/ high-density lipoprotein cholesterol $<3.1 / \geq 1.0 \mathrm{mmol} / \mathrm{L}$ and non-high-density lipoprotein cholesterol $<3.8 \mathrm{mmol} / \mathrm{L}$ were investigated.

Results: The rates of achieving targets for $\mathrm{HbA1c}$, blood pressure, and lipids were $52.9 \%, 46.8 \%$ and $65.5 \%$, respectively. The prevalence of microvascular complications was $28 \%$ each, $6.4 \%$ of which had all microvascular complications, while that of macrovascular complications was $12.6 \%$. With an increasing duration of diabetes, the rate of achieving target $\mathrm{HbA} 1 \mathrm{c}$ decreased and the prevalence of each complication increased despite increased use of diabetes medication. The prevalence of each complication decreased according to the number achieving the 3 treatment targets and was lower in subjects without macrovascular complications than those with. Adjustments for considerable covariates exhibited that each complication was closely interrelated, and the achievement of each target was significantly associated with being free of each complication.

Conclusions: Almost half of the subjects examined did not meet the recommended targets. The risk of each complication was significantly affected by 1 ontarget treatment (inversely) and the concomitance of

\section{Key messages}

- The up-to-date rates of achieving treatment targets and prevalence of complications were surveyed in this study and shown according to the duration of diabetes.

- Almost half of the subjects did not meet the recommended targets, and especially the rate of achieving the $\mathrm{HbA1C}$ target markedly decreased with longer durations despite increases in the use of any diabetes medication.

- Excess bodyweight was associated with deleterious effects on achieving therapeutic targets and prevalence of complications, especially of nephropathy.

another complication (directly). Total diabetes care including one-by-one management of modifiable risk factors and complications may be important for high-quality care. The future studies including more subjects and clinics with precise complication status are needed.

\section{INTRODUCTION}

With the expected increase in the prevalence of type 2 diabetes mellitus (T2DM) in the population, due in part to increasing rates of obesity and decreased physical activity, the burden of microvascular complications, known as diabetic nephropathy, retinopathy, and neuropathy, may also increase. ${ }^{1-3}$ Diabetic nephropathy is the most common single cause of end-stage renal disease in the USA, Europe, and Japan. ${ }^{4}$ It is also the leading cause of blindness among working-aged adults worldwide. ${ }^{5}$ Diabetic neuropathy is associated with considerable morbidity, mortality, and diminished quality of life. ${ }^{6}$ The estimates of the prevalence of microvascular complications, as well as macrovascular complications, and their relationships with major 
risk factors are crucial for guiding public health education and the optimal management of diabetes. The prevalence of microvascular complications has been extensively examined; however, most studies have only referred to a single microvascular complication. Few studies have investigated the three microvascular complications simultaneously in the same subjects at the same time point. The prevalence of the overlap of two or three microvascular complications, and its association with macrovascular complications, are important for estimating the future risk of other complications and mortality; however, these issues have not yet been investigated in detail until now.

On the other hand, quality of care for individuals with T2DM may have improved in the past two decades, ${ }^{3}$ 7-9 reflecting recent advances in treatments for blood glucose, blood pressure (BP), and lipids. However, the bodyweight of subjects with T2DM is increasing ${ }^{70}$ and excess bodyweight is a significant risk factor for increased morbidity and mortality. ${ }^{10}$ The adverse effects of excess bodyweight on the management of blood glucose, BP and lipids, as well as on the prevalence of microvascular/macrovascular complications in T2DM, have not been sufficiently elucidated. Therefore, it is important to obtain the up-to-date rates of achieving therapeutic targets in association with bodyweight levels and their relationships with diabetic microvascular complications as well as macrovascular complications. The aim of the present study is to investigate the prevalence of three microvascular complications in the same subject with T2DM at the same time, and simultaneously examine the rates of achieving targets in glycated hemoglobin (HbA1c), BP, and lipids through an updated large-scale multicenter-based cohort. Cross-sectional associations between therapeutic target achievements and microvascular/macrovascular complications in association with bodyweight were also examined.

\section{METHODS}

\section{Study population}

Seventeen medical clinics (ie, general practitioners (GPs) specializing in diabetes care) volunteered to participate in this study. These clinics were located in different areas in Japan and used the same software to incorporate patient records in the Japan Diabetes Clinical Data Management (JDDM) Study Group. ${ }^{11}$ The group consists of medical doctors who have dedicated their time to recording clinical data of their patients during daily clinical practice for scientific analyses. The study was performed in primary care settings. Subjects were at first enrolled from those who consecutively attended these clinics in 2013 and had urinary albumin and serum creatinine $(\mathrm{Cr})$ measured within a year $(\mathrm{N}=13039)$. Among them, subjects who had retinopathy and neuropathy evaluated within a year were registered in this study $(\mathrm{N}=9956)$. This covered about $20 \%$ of subjects attending the clinics in the JDDM study group. Subjects were treated with the aim of achieving the targets recommended by the Japan Diabetes Society (JDS) of a
HbAlc value of $<7.0 \% \quad(53 \mathrm{mmol} / \mathrm{mol}), \quad \mathrm{BP}<130 /$ $80 \mathrm{~mm} \mathrm{Hg}$, and serum concentrations of low-density lipoprotein (LDL) cholesterol $<3.1 \mathrm{mmol} / \mathrm{L} \quad(120 \mathrm{mg} / \mathrm{dL})$, high-density lipoprotein (HDL) cholesterol $\geq 1.0 \mathrm{mmol} / \mathrm{L}$ $(40 \mathrm{mg} / \mathrm{dL}),{ }^{12}$ and non-HDL cholesterol $<3.8 \mathrm{mmol} / \mathrm{L}$ $(150 \mathrm{mg} / \mathrm{dL})$. The most common treatment practices during the study period included dipeptidyl peptidase- 4 inhibitors with metformin for blood glucose, angiotensin II receptor blockers for $\mathrm{BP}$, and statins for lipids. The study protocol was approved by the Ethics Committees of the JDDM. All patients provided informed consent and the study was performed in accordance with Helsinki Declaration II.

\section{Measurements}

$\mathrm{BP}$ was measured with an appropriately sized cuff in the sitting position after resting for at least $5 \mathrm{~min}$, using an automated standardized BP device. Non-fasting blood samples were drawn and analyzed to measure serum $\mathrm{Cr}$ and lipids at local laboratories. HbA1c was measured by high-performance liquid chromatography, which has been certified by the American National Glycohemoglobin Standardization Program. Serum and urinary concentrations of $\mathrm{Cr}$ were measured by an enzymatic method. Urinary albumin was measured using random urine samples, in the absence of menstruation and urinary tract infection, by a turbidimetric immunoassay. The urinary albumin excretion rate was recorded as the albumin-to-creatinine ratio (ACR). Normoalbuminuria, microalbuminuria, and macroalbuminuria were defined as $\mathrm{ACR}<30, \mathrm{ACR} \geq 30$ and $<300$, and $\mathrm{ACR} \geq 300 \mathrm{mg} / \mathrm{g} \mathrm{Cr}$, respectively. Glomerular filtration rate (GFR) was estimated using the following equation by the Japanese Society of Nephrology: estimated glomerular filtration rate $(\mathrm{eGFR}) \quad\left(\mathrm{mL} / \mathrm{min} / 1.73 \mathrm{~m}^{2}\right)=194 \times$ serum creatinine $(\mathrm{Scr})^{-1.094} \times \mathrm{Age}^{-0.287} \times 0.739$ (if female). ${ }^{13}$ Chronic kidney disease (CKD) was defined by $\mathrm{ACR} \geq 30 \mathrm{mg} / \mathrm{g} \mathrm{Cr}$ or eGFR $<60 \mathrm{~mL} / \mathrm{min} / 1.73 \mathrm{~m}^{2}$. LDL cholesterol was calculated by Friedewald's formula. Regarding the treatment of diabetes, subjects were divided into groups treated by diet alone, hypoglycemic tablets, tablets with insulin, and only insulin. Macrovascular complications, that is, cardiovascular diseases (CVD) of coronary artery disease (CAD), ischemic stroke, and peripheral artery disease (PAD), including coronary, cerebrovascular, and carotid revascularization, were noted. PAD was diagnosed when intermittent claudication occurred, with the confirmation of an ankle-brachial pressure index $<0.9$ or significant peripheral artery stenosis by angiography, or leg amputation above the ankle as a result of diabetes.

Subjects attended the clinic every 1-2 months. This prevalence study focused on the presence of microvascular complications and conditions related to diabetes in the early stages. In order to assess diabetic retinopathy, subjects were referred to an eye clinic at least once a year. Diabetic retinopathy was diagnosed after pupillary dilation by an ophthalmologist using ophthalmoscopy. According to the Early Treatment Diabetic Retinopathy 
Study grading standards, ${ }^{14}$ background retinopathy was defined as the presence of one or more retinal microaneurysms or retinal blot hemorrhages and proliferative retinopathy was diagnosed as the presence of new vessels in the retina or vitreous hemorrhage. In order to assess diabetic peripheral neuropathy, subjects underwent an annual physical examination. Diabetic neuropathy, which is referred to as diabetic symmetrical sensorimotor polyneuropathy, diabetic polyneuropathy, or diabetic peripheral neuropathy, was diagnosed in patients with two or more of three components, as recommended in the simplified diagnostic criteria proposed by the Diabetic Neuropathy Study Group in Japan: ${ }^{15}$ (1) subjective symptoms in the bilateral lower limbs or feet; (2) loss of or decreased ankle jerk reflex and (3) decreased vibration perception, assessed using a C128 tuning fork and bilaterally measured at the medial malleoli.

\section{Statistical analysis}

Data were expressed as the mean \pm SD if normally distributed. The significance of differences between groups was assessed by $\chi^{2}$ tests for categorical variables and analysis of variance (ANOVA) for continuous variables. A multivariate logistic regression analysis was performed to investigate the clinical variables associated with the presence of nephropathy, retinopathy, neuropathy, and CVD. The covariates were sex, age, duration of diabetes, body mass index (BMI), HbA1c on target, BP on target, lipids on target, and eGFR in model A. Model B additionally included nephropathy, retinopathy, and neuropathy. A p value of $<5 \%$ (two-tailed) was considered significant. All analyses were performed with the statistical software package SPSS (SPSS Japan, Tokyo, Japan).

\section{RESULTS}

\section{Rates of achieving treatment target and prevalence of}

\section{microvascular and macrovascular complications}

The clinical characteristics of subjects with T2DM are shown in table 1 . Of the 9956 subjects with an average age of 65 years, duration of 14 years, BMI of $24.9 \mathrm{~kg} / \mathrm{m}^{2}$, $\mathrm{BP}$ of $126 / 72 \mathrm{~mm} \mathrm{Hg}$, and HbAlc of $7.04 \%$, 29.5\% had nephropathy including $23.9 \%$ with microalbuminuria and $5.6 \%$ with macroalbuminuria, $26.4 \%$ had any retinopathy, and $27.7 \%$ had neuropathy. According to the duration of diabetes, the proportions of the use of insulin, antihypertensive and lipid-lowering agents, albuminuria, retinopathy, neuropathy, and macrovascular complications of CAD and stroke increased significantly. The values of HbA1c, systolic BP, HDL cholesterol, and serum $\mathrm{Cr}$ increased with a longer duration of diabetes, whereas the values of BMI, diastolic BP, LDL cholesterol, non-HDL cholesterol, and eGFR decreased. According to the duration, the rate of achieving HbAlc target decreased significantly, whereas the rate for BP was unchanged and that for lipids increased.

Figure 1 shows the rates of achieving the targets for HbA1c, BP, and lipids (figure 1A) and prevalence of microvascular complications (figure 1B) in the 9956 subjects. A total of $20.8 \%$ achieved all three targets, whereas $11.8 \%$ achieved none of the targets. Among all subjects, $6.4 \%$ had all three microvascular complications, whereas 45.7\% had none. The relationships between achieving treatment targets with the presence/absence of each complication are shown in online supplementary table S1. A clear correlation was found between achieving each target and the absence of each complication except for lipids with retinopathy.

Figure 2 indicates the prevalence of the three microvascular complications by the number of achievements out of the three treatment targets (figure 2A-D) and between subjects with and without CVD (figure 2E, F). The proportion free from any complication was highest at $53.2 \%$ in subjects achieving three treatment targets, and decreased step by step, reaching $34.1 \%$ in subjects achieving no targets $\left(\chi^{2}\right.$ score $\left.179.4, \mathrm{p}<0.001\right)$. In contrast, the prevalence of all three microvascular complications was the lowest at $3.5 \%$ in subjects achieving three treatment targets, and increased to $11.1 \%$ in subjects achieving no targets $\left(\chi^{2}\right.$ score $\left.89.9, \mathrm{p}<0.001\right)$. Subjects with CVD evidently had a higher prevalence of nephropathy, retinopathy, and neuropathy than those without CVD ( $\mathrm{p}<0.001$, respectively).

\section{Effects of BMII on microvascular and macrovascular complications}

The relationships of BMI levels with achieving targets for HbAlc, BP, and lipids, and prevalence of nephropathy, retinopathy, and neuropathy are shown in figure 3 . Increasing BMI levels correlated with decreasing rates of all three target achievements $(\mathrm{p}<0.001$, respectively). The prevalence of nephropathy and retinopathy increased significantly with higher BMI levels $\left(\chi^{2}\right.$ score $122.0, \mathrm{p}<0.001 ; \chi^{2}$ score $15.6, \mathrm{p}<0.01$, respectively), whereas neuropathy was not associated with BMI levels ( $\chi^{2}$ score 4.7 , not significant).

\section{Risk analyses for microvascular and macrovascular complications}

We investigated whether the levels of albuminuria and eGFR are associated with the prevalence of retinopathy, neuropathy, and CVD, and the results obtained are shown in online supplementary table S2. The higher levels of albuminuria and lower levels of eGFR significantly increased the prevalence rates of retinopathy, neuropathy, and CVD. To explore variables associated with nephropathy, retinopathy, neuropathy, and CVD, multivariate logistic regression analyses were performed, as shown in table 2. Regarding microvascular complications, achieving treatment targets for $\mathrm{HbAlc}$ and $\mathrm{BP}$ were significantly associated with nephropathy and retinopathy independent of the effects of sex, age, duration, BMI, and eGFR, whereas achieving the treatment targets for lipids was significantly associated only with nephropathy and neuropathy. Regarding CVD, nephropathy, retinopathy, and neuropathy were independently associated 


\begin{tabular}{|c|c|c|c|c|}
\hline & \multirow[b]{2}{*}{ Total } & \multicolumn{3}{|c|}{ Duration of diabetes } \\
\hline & & $<10$ years & 10 to $<20$ years & $\geq 20$ years \\
\hline $\mathrm{N}$ (male \%) & 9956 (62.2) & $3580(62.6)$ & 3919 (61.5) & 2457 (62.8) \\
\hline Age, years & $65 \pm 12$ & $61 \pm 12$ & $66 \pm 11$ & $71 \pm 9^{\star \star \star}$ \\
\hline Duration of diabetes, years & $14 \pm 9$ & $6 \pm 3$ & $14 \pm 3$ & $27 \pm 6^{\star \star \star}$ \\
\hline $\mathrm{BMI}, \mathrm{kg} / \mathrm{m}^{2}$ & $24.9 \pm 4.2$ & $25.5 \pm 4.3$ & $25.0 \pm 4.2$ & $24.0 \pm 3.9^{\star \star \star}$ \\
\hline $\mathrm{HbA} 1 \mathrm{c}, \%$ & $7.04 \pm 0.89$ & $6.91 \pm 0.92$ & $7.10 \pm 0.90$ & $7.16 \pm 0.81^{\star \star \star}$ \\
\hline Diet/tablets/tablets+insulin/insulin (\%) & 12.1/69.3/14.1/4.5 & 20.5/69.8/6.6/3.1 & 8.6/74.4/13.6/3.5 & $5.4 / 60.4 / 25.9 / 8.3^{\star \star \star}$ \\
\hline $\mathrm{HbA} 1 \mathrm{c}$ on target $<7.0 \%, \mathrm{~N}(\%)$ & $5268(52.9)$ & $2213(61.8)$ & $19629(50.1)$ & $1093(44.5)^{\star \star \star}$ \\
\hline Systolic/diastolic BP, mm Hg & $126 \pm 14 / 72 \pm 11$ & $126 \pm 14 / 74 \pm 10$ & $127 \pm 14 / 72 \pm 10$ & $127 \pm 15^{\star \star \star} / 69 \pm 11^{\star \star \star}$ \\
\hline Use of antihypertensive agents, N (\%) & $5308(53.3)$ & $1586(44.3)$ & $2149(54.8)$ & $1573(64.0)^{\star \star \star}$ \\
\hline Hypertension, N (\%) & $5945(59.7)$ & $1884(52.6)$ & $2368(60.4)$ & $1693(68.9)^{\star * *}$ \\
\hline BP on target $<130 / 80 \mathrm{~mm} \mathrm{Hg}, \mathrm{N}(\%)$ & $4656(46.8)$ & $1703(47.6)$ & $1829(46.7)$ & $1124(45.7)$ \\
\hline LDL cholesterol, mg/dL & $105 \pm 27$ & $109 \pm 29$ & $105 \pm 27$ & $102 \pm 26^{\star \star \star}$ \\
\hline HDL cholesterol, mg/dL & $55.3 \pm 14.9$ & $54.8 \pm 14.6$ & $55.3 \pm 15.0$ & $55.9 \pm 15.2^{*}$ \\
\hline Non-HDL cholesterol, mg/dL & $130 \pm 31$ & $135 \pm 33$ & $130 \pm 31$ & $124 \pm 28^{\star \star \star}$ \\
\hline Use of lipid-lowering agents, N (\%) & $4661(46.8)$ & $1538(43.0)$ & $1942(49.6)$ & $1181(48.1)^{\star \star \star}$ \\
\hline Dyslipidemia, N (\%) & 7104 (71.4) & $2563(71.6)$ & $2864(73.1)$ & $1677(68.3)^{\star \star \star}$ \\
\hline Lipids on target, $\mathrm{N}(\%)$ & $6524(65.5)$ & $2165(60.5)$ & $2593(66.2)$ & $1766(71.9)^{\star \star \star}$ \\
\hline All (HbA1c, BP, lipids) on target, N (\%) & $2070(20.8)$ & $813(22.7)$ & $795(20.3)$ & $462(18.8)^{\star *}$ \\
\hline Serum creatinine, $\mathrm{mg} / \mathrm{dL}$ & $0.83 \pm 0.45$ & $0.78 \pm 0.34$ & $0.82 \pm 0.38$ & $0.92 \pm 0.65^{\star \star \star}$ \\
\hline eGFR, $\mathrm{ml} / \mathrm{min} / 1.73 \mathrm{~m}^{2}$ & $71 \pm 19$ & $76 \pm 19$ & $71 \pm 19$ & $65 \pm 19^{\star \star \star}$ \\
\hline \multicolumn{5}{|l|}{ Albuminuria } \\
\hline Normoalbuminuria, N (\%) & 7017 (70.5) & $2738(76.5)$ & $2728(69.6)$ & $1551(63.1)^{\star \star \star}$ \\
\hline Albuminuria, $\mathrm{N}(\%)$ & 2939 (29.5) & $842(23.5)$ & $1191(30.4)$ & $906(36.9)^{\star \star \star}$ \\
\hline Microalbuminuria, N (\%) & $2376(23.9)$ & $693(19.4)$ & $985(25.1)$ & $698(28.4)^{\star \star \star}$ \\
\hline Macroalbuminuria, $\mathrm{N}(\%)$ & $563(5.6)$ & $149(4.2)$ & $206(5.3)$ & $208(8.5)^{\star \star \star}$ \\
\hline CKD, N (\%) & $4431(44.5)$ & $1251(34.9)$ & $1779(45.4)$ & $1401(57.0)^{\star \star \star}$ \\
\hline \multicolumn{5}{|l|}{ Retinopathy } \\
\hline None, N (\%) & $7328(73.6)$ & $3048(85.1)$ & $2881(73.5)$ & $1399(56.9)^{\star \star \star}$ \\
\hline Any retinopathy, N (\%) & $2628(26.4)$ & $532(14.9)$ & $1038(26.5)$ & $1058(43.1)^{\star \star \star}$ \\
\hline Background, N (\%) & 1489 (15.0) & $304(8.5)$ & $643(16.4)$ & $542(22.1)^{\star \star \star}$ \\
\hline Proliferative, $\mathrm{N}(\%)$ & 1139 (11.4) & $228(6.4)$ & $395(10.1)$ & $516(21.0)^{\star \star \star}$ \\
\hline \multicolumn{5}{|l|}{ Neuropathy } \\
\hline Abnormality $\geq 2$ of the below a-c, $N(\%)$ & $2760 / 9956(27.7)$ & $695 / 3580(19.4)$ & $1119 / 3919(28.6)$ & $946 / 2457(38.5)^{\star \star \star}$ \\
\hline $\mathrm{a} / \mathrm{b} / \mathrm{c}(\%)$ & 22.3/44.6/36.2 & $17.1 / 33.9 / 29.7$ & $22.3 / 46.3 / 36.8$ & $29.9^{\star \star \star} / 57.0^{\star \star \star} / 45.0^{\star \star \star}$ \\
\hline All microvascular complications, N (\%) & $639(6.4)$ & $120(3.4)$ & $232(5.9)$ & $287(11.7)^{\star \star \star}$ \\
\hline Macrovascular complications, N (\%)† & $1234 / 9809(12.6)$ & $270 / 3515(7.7)$ & $476 / 3856$ (12.3) & $488 / 2438(20.0)^{\star \star \star}$ \\
\hline CAD, N (\%) & $686(7.0)$ & $122(3.5)$ & $269(7.0)$ & $295(12.1)^{\star \star \star}$ \\
\hline Ischemic stroke, N (\%) & $600(6.2)$ & $138(4.0)$ & $229(6.0)$ & $233(9.6)^{\star \star \star}$ \\
\hline PAD, N (\%) & $116(1.3)$ & $38(1.2)$ & $39(1.1)$ & $39(1.7)$ \\
\hline
\end{tabular}

a, Symptoms of polyneuropathy; $b$, abnormal tendon reflex; $c$, abnormal vibration perception.

${ }^{\star} p<0.05,{ }^{* \star} p<0.01$, and ${ }^{\star \star *} p<0.0001$ by a one-way ANOVA for continuous variables and $\chi^{2}$ test for categorical variables.

†Data for cardiovascular diseases were available in 9809 subjects.

ANOVA, analysis of variance; BMI, body mass index; BP, blood pressure; CAD, coronary artery disease; CKD, chronic kidney disease; eGFR, estimated glomerular filtration rate; HbA1c, glycated hemoglobin; HDL, high-density lipoprotein; LDL, low-density lipoprotein; PAD, peripheral artery disease; T2DM, type 2 diabetes mellitus.

with CVD after adjustments for the effects of sex, age, duration, BMI, and eGFR, whereas achieving targets for $\mathrm{BP}$ and lipids were not.

\section{DISCUSSION}

This study provides the latest estimates of the prevalence of nephropathy, retinopathy, and neuropathy in association with putative risk factors including achievement of the treatment targets for HbAlc, BP, and lipids, CVD, and weight excess, for subjects with T2DM attending
GPs in the JDDM study in Japan. In a large-scale multicenter-based survey conducted in 2013, we found that $52.9 \%$ achieved HbAlc $<7.0 \%, 46.8 \%$ achieved $\mathrm{BP}<130 / 80 \mathrm{~mm} \mathrm{Hg}$, and $65.5 \%$ achieved the target for lipids, which yielded $20.8 \%$ that met all three treatment targets and $11.8 \%$ that achieved none of the targets. On the other hand, the prevalence of nephropathy, retinopathy, and neuropathy was $~ 30 \%$ each, $6.4 \%$ of which had all three microvascular complications and $45.7 \%$ had no microvascular complications. Notably, when a subject had a complication, the overlap rates of other 
A Rates of achieving target of HbA1c, BP, and lipids

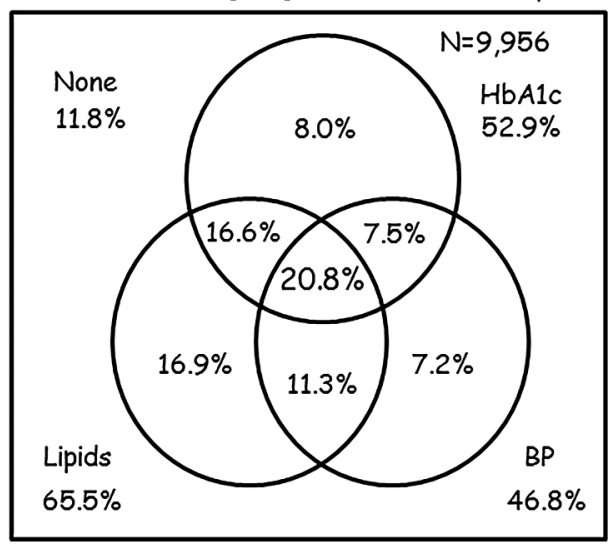

B Prevalence of microvascular complications

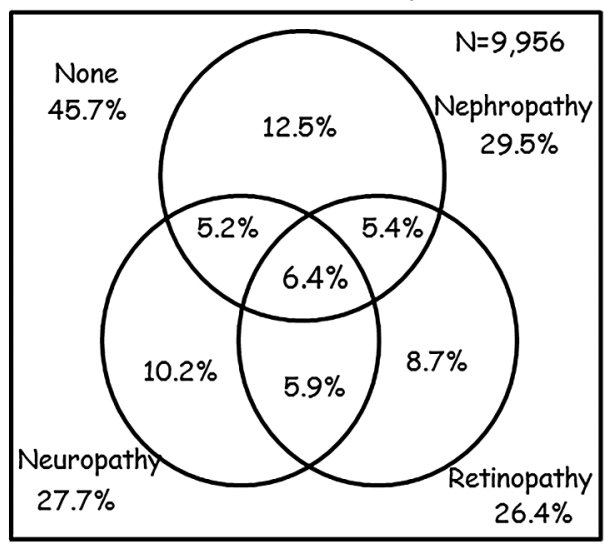

Figure 1 (A) Rate of achieving targets for glycated hemoglobin ( $\mathrm{HbA} 1 \mathrm{c})$, blood pressure (BP), and lipids in all subjects and (B) prevalence of nephropathy, retinopathy, and neuropathy in subjects with type 2 diabetes mellitus $(n=9956)$.
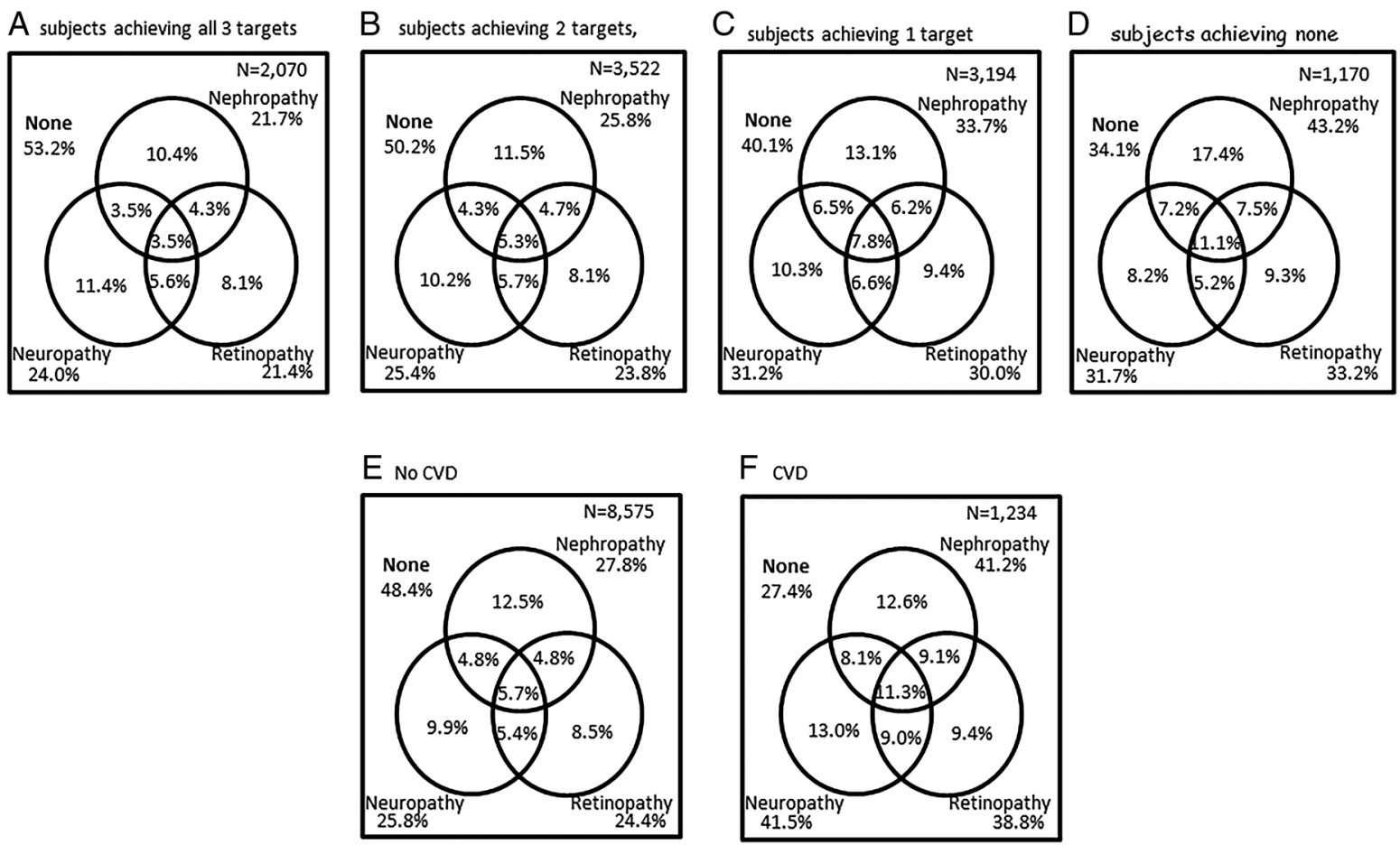

Figure 2 Prevalence of nephropathy, retinopathy, and neuropathy in subjects achieving all (A) three targets, (B) two targets, (C) one target, and (D) none, and in subjects (E) without cardiovascular diseases (CVD) and (F) with CVD.

microvascular complications were high; for instance, out of subjects with retinopathy, $67 \%(17.7 / 26.4 \%)$ had other microvascular complications. This was prominent when subjects had CVD. The prevalence of microvascular as well as macrovascular complications became higher with increases in the duration of T2DM. Importantly, the rate of achieving the HbAlc target markedly decreased with longer durations despite increases in the use of any diabetes medication (tablets or insulin), whereas the rate of achieving the BP target remained unchanged. The rate of achieving the lipids target increased with longer durations; this may partly be explained by the lower BMI and higher use of lipid-lowering agents.

The proportions that met the treatment targets in this study are similar to those reported among 1444 subjects with adult diabetes from the National Health and Nutrition Examination Survey (NHANES) data surveyed in $2010 .{ }^{9}$ The findings showed $52.2 \%$ with $\mathrm{HbA1c}<7.0 \%$, $51.3 \%$ with $\mathrm{BP}<130 / 80 \mathrm{~mm} \mathrm{Hg}, 56.8 \%$ with LDL cholesterol $<130 \mathrm{mg} / \mathrm{dL}$, and $14.3 \%$ that met all three targets. Regarding the prevalence of microvascular complications, the JDDM study reported that those of normoalbuminuria/albuminuria were $69.7 / 30.3 \%$ in $2004,{ }^{16}$ which 
A $B M I<25$

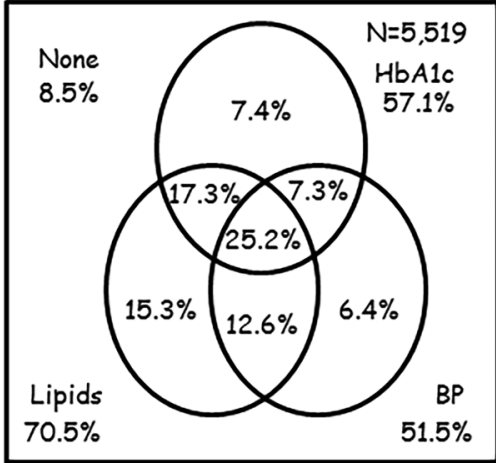

$D B M I<25$

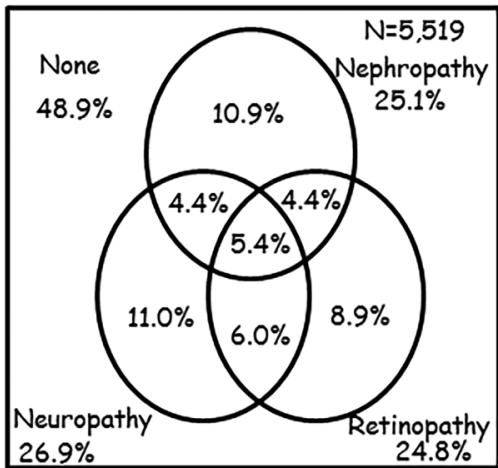

B BMI 25- $<30$

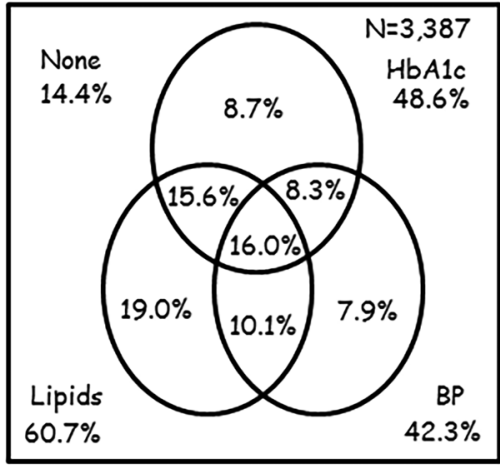

E BMI 25- $<30$

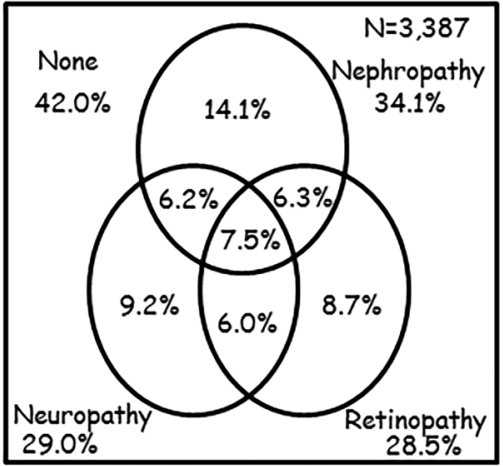

C $B M I \geq 30$

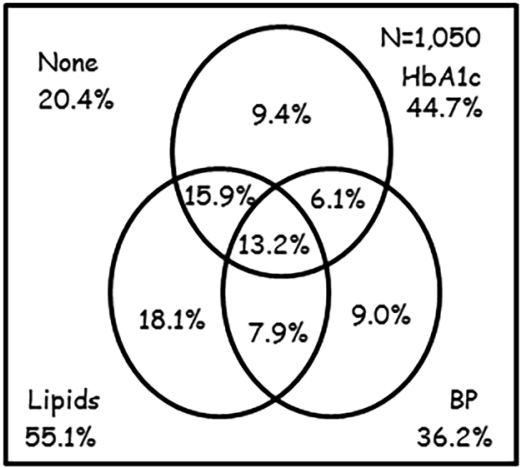

F $\quad B M I \geq 30$

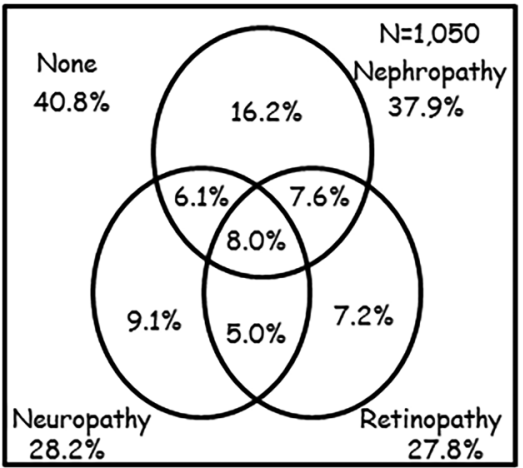

Figure 3 Associations of body mass index (BMI) levels with achieving targets for glycated hemoglobin (HbA1c), blood pressure (BP), and lipids in the upper panels $A, B$, and $C$, and with the prevalence of nephropathy, retinopathy, and neuropathy in the lower panels of $\mathrm{D}, \mathrm{E}$, and $\mathrm{F}$.

were similar to the present survey. There have been many studies on the prevalence of retinopathy. Previous studies, with the number of subjects with T2DM being $\sim 10000$ or more and published in the past decade, showed the prevalence of any retinopathy to be $30.3 \%$ (26 216/86 390) in Wales, ${ }^{17} 16.3 \%$ (8584/52 736) in Portugal, ${ }^{18} 12.3 \% \quad(13387 / 108723)$ in Catalonia, ${ }^{19}$ $19.3 \%(8409 / 43523$ in newly diagnosed T2DM) in Scotland, ${ }^{20}$ and $25.3 \%$ (16 094/63 622) in Norfolk. ${ }^{21}$ Compared with the studies on retinopathy, there have been fewer studies on the prevalence of neuropathy, and the number of subjects examined was also smaller. The previous reports showed the prevalence of neuropathy to be $29.2 \%(586 / 2006)$ in India, ${ }^{22} 30.5 \%(1100 / 3591)$ in Verona, ${ }^{23} 33.1 \%(1113 / 3359)$ in China, ${ }^{24}$ and $33.5 \%$ $(1338 / 4000)$ in Korea. ${ }^{25}$ The prevalence rates described above were subject to population characteristics such as the duration of diabetes, ethnicity, and risk factors including blood glucose and BP control; however, these factors were not sufficiently considered in the above studies and referred to only a single microvascular complication.

In this cross-sectional design, we found strong relationships between achieving targets for HbAlc, BP and lipids and being free of nephropathy, retinopathy, and neuropathy. After adjustments for gender, age, duration, $\mathrm{BMI}$, and eGFR in the multivariate logistic regression analysis, achieving each target independently correlated (inversely) with nephropathy, retinopathy, and neuropathy, except for the effect of lipids control on retinopathy. This result appears to support glycemic control as well as BP and lipid controls, being essential for preventing the three microvascular complications. Furthermore, we found that the number achieving the treatment targets correlated inversely with the prevalence of microvascular complications. This result indicates the importance of achieving treatment targets one by one. These inhibitory effects were not observed with CVD; the reason for this may be that $\mathrm{BP}$ and lipids are aimed at stricter levels than before the onset of CVD. Nevertheless, HbAlc on target correlated with being free of CVD. The absence of a relationship between lipids and retinopathy was supported by other studies, in which serum lipids were associated with severe macular edema but not with retinopathy. ${ }^{26}{ }^{27}$ The underlying mechanism suggested that lipid extravasation is restricted in the retina by the blood-retinal barrier and disruption of this barrier, rather than increased lipid levels, is required for lipid-induced retinal injury. ${ }^{28}$

Higher BMI levels were associated with the reduction of the rates of achieving therapeutic targets for HbAlc, BP, and lipids. This deleterious impact of excess bodyweight on blood glucose, BP, and lipids is consistent with the concept that excess bodyweight is now the sixth most important risk factor contributing to the overall burden 


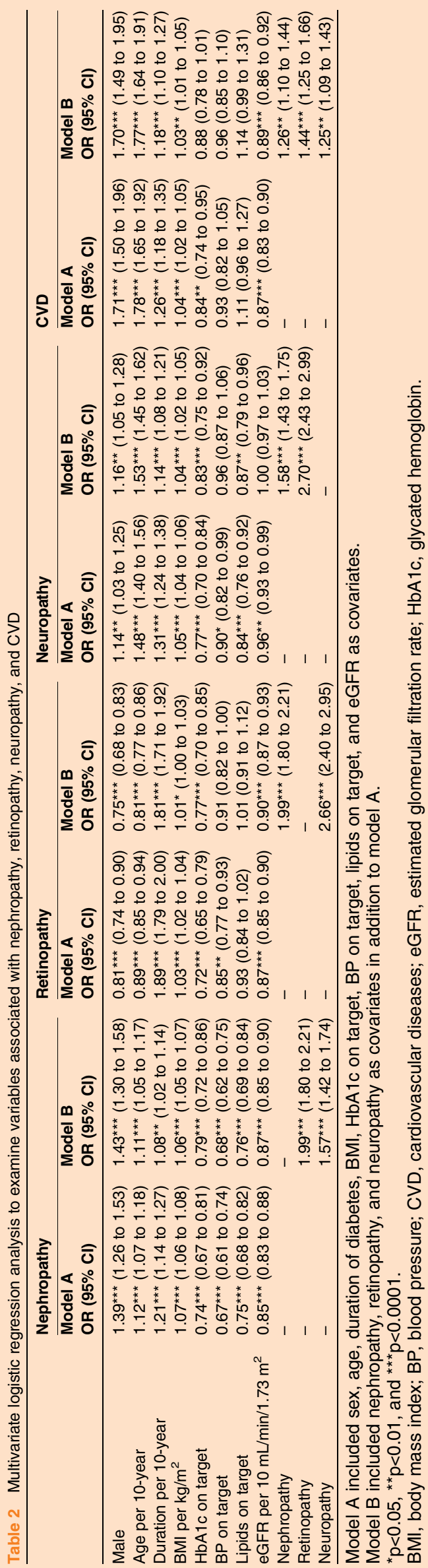

of diseases worldwide. ${ }^{29} 30$ The pathological mechanisms underlying obesity-related resistance to each treatment include insulin resistance in the nitric oxide pathway, the stimulatory effects of hyperinsulinemia on sympathetic drive, smooth muscle growth, and sodium-fluid retention, and increased angiotensin II. $^{31}{ }^{32}$ The smaller number of achieving therapeutic targets in subjects with higher BMI may reflect the increasing prevalence of nephropathy and retinopathy. The marked increase observed in the prevalence of nephropathy may be associated with the incremental involvement of obesity-related kidney disease, in which increased angiotensin II activity is suggested to play a central role in hypertension, dyslipidemia, and insulin resistance. ${ }^{32}$ BMI per se was found to be an independent risk of nephropathy, retinopathy, and neuropathy in the present multiple logistic regression analyses. This may suggest a causal role of higher BMI in the pathogenesis of microvascular complications that should be investigated in the future.

This study identified close and strong links as well as inter-relationships between each complication, that is, one microvascular complication was closely related to another microvascular complication and a macrovascular complication. Furthermore, the elevated albuminuria and decreased eGFR levels were closely related to retinopathy, neuropathy, and macrovascular complications. This cross-sectional study is unable to refer to the causal relationship; however, these results would indicate that one microvascular complication or abnormal levels in albuminuria or eGFR strongly increase the risk of developing other microvascular and macrovascular complications. Several reports support our findings of the close association of retinopathy with albuminuria, ${ }^{33} 34$ GFR, ${ }^{34}{ }^{35}$ and $\mathrm{CVD},{ }^{36}$ and that of CVD with albuminuria and GFR, ${ }^{37} 38$ whereas few studies described the association of neuropathy with albuminuria and GFR. ${ }^{39}$ Our findings were supported by a recent study which indicated the clear associations of the number of microvascular complications with the onset of CVD in the prospective manner. ${ }^{40}$ Endothelial cells, if exposed to chronic hyperglycemia, develop intracellular hyperglycemia, because they cannot downregulate glucose transport. $^{41}$ A common pathological trait of diabetic microvascular disease is progressive narrowing and eventual occlusion of vascular lumina, ${ }^{42}$ and hyperglycemia may trigger and initiate endothelial dysfunction leading to macrovascular disease. ${ }^{43}$ Taken together, it is important to emphasize that multifactorial treatments for and the assessment of each complication including albuminuria and GFR are essential for high-quality diabetes care.

Our study is subject to several limitations. First, a selection bias may have been caused by participating GPs specializing in diabetes care that influenced the results; the prevalence might be lower with a higher rate of achieving treatment targets than in real-world practice. In contrast, a possibility that severely complicated subjects were referred to these participating GPs because they were specializing in diabetes care cannot be excluded, which 
might have led to worse outcomes. For subjects with severe complications, the GPs consult with cardiologists, nephrologists, ophthalmologists, and specialists at tertiary care centers, but mostly continue to take care of the patients depending on the severity, even after discharging the hospitals. Second, we should acknowledge that we did not extensively investigate severity in terms of proliferative retinopathy and autonomic neuropathy because we focused on the presence of a microvascular complication and conditions related to diabetes in their early stages. Third, analyses were restricted to individuals in whom complete information was available on prevalent microvascular diseases, and thus may have been subject to a selection bias. An examination of the rates of achieving treatment target and prevalence of complications, as well as the association between risks and microvascular/ macrovascular diseases, among individuals with data missing on all three diseases showed no qualitative difference with the complete cohort. Fourth, macrovascular complication data were missing in 147 subjects (1.5\%), which was trivial. The strength of this survey is that it was based on patient records provided by physicians, which is more accurate than self-reported data from patients. In the absence of a nationally registered system in Japan, this largest multicenter-based study is likely to be representative of subjects with T2DM.

In conclusion, almost half of the subjects did not meet the recommended targets. In the absence of a nationally registered system in Japan, this largest survey is likely to represent and provide a robust estimate of the national target achieving rates and prevalence of complications in T2DM. The risk of each complication was significantly affected by one on-target treatment (inversely) and the concomitance of another complication (directly). Total diabetes care including one-by-one management of modifiable risk factors and complications may be important for highquality care. The future studies including more subjects and clinics with precise complication status are needed.

\author{
Author affiliations \\ ${ }^{1}$ Department of Internal Medicine, Jiyugaoka Medical Clinic, Obihiro, Japan \\ ${ }^{2}$ Oishi Clinic, Kyoto, Japan \\ ${ }^{3}$ Takamura Clinic, Fussa, Japan \\ ${ }^{4}$ Kawai Clinic, Tsukuba, Japan \\ ${ }^{5} \mathrm{HEC}$ Science Clinic, Yokohama, Japan \\ ${ }^{6}$ Hotaruno Central Clinic, Kisarazu, Japan \\ ${ }^{7}$ Sugimoto Clinic, Kitakyushu, Japan \\ ${ }^{8}$ Kurihara Clinic, Sapporo, Japan \\ ${ }^{9}$ Department of Medicine, Shiga University of Medical Science, Otsu, Shiga, \\ Japan
}

Acknowledgements Ms Sanae Horiguchi-Watanabe and Mr Suguho Takahashi ( Jiyugaoka Medical Clinic) are thanked for their skilful data collection and analyses. The following clinics from the JDDM study group contributed to this study: Fukumoto Clinic (Kagoshima), HEC Science Clinic (Kanagawa), Hikari Clinic (Nara), Hotaruno Central Clinic (Chiba), lizumi Clinic (Ibaraki), Iwasaki Clinic (Yamaguchi), Jiyugaoka Medical Clinic (Hokkaido), Kawai Clinic (Ibaraki), Kurihara Clinic (Hokkaido), Mirai Kunitachi Clinic (Tokyo), Oishi Clinic (Kyoto), Okuguchi Clinic (Miyagi), Sugimoto Clinic (Fukuoka), Takai Clinic (Kanagawa), Takamura Clinic (Tokyo), Tama Center Mirai Clinic (Tokyo), Wakamatsu Memorial Hospital (Kagoshima), and Yagi Clinic (Okinawa).
Contributors HY, MO, HT, and YK designed the study. HY generated data in collaboration with the study group office. HY and SA performed statistical analyses. All authors contributed to the interpretation of the data and drafting of the manuscript. HY takes responsibility for statistical method and organized for performing multivariate analysis. All authors critically reviewed the manuscript. $\mathrm{HY}$ is the guarantor of this work and, as such, had full access to all the data in the study and takes responsibility for the integrity of the data and the accuracy of the data analysis.

Competing interests None declared.

Ethics approval The study protocol was approved by the Ethics Committees of the JDDM.

Provenance and peer review Not commissioned; externally peer reviewed.

Data sharing statement Data are shared by HY, MO, HT, KY, SS, DU, HS, YK, and the study group office.

Open Access This is an Open Access article distributed in accordance with the Creative Commons Attribution Non Commercial (CC BY-NC 4.0) license, which permits others to distribute, remix, adapt, build upon this work non-commercially, and license their derivative works on different terms, provided the original work is properly cited and the use is non-commercial. See: http://creativecommons.org/licenses/by-nc/4.0/

\section{REFERENCES}

1. NCD Risk Factor Collaboration (NCD-RisC). Worldwide trends in diabetes since 1980: a pooled analysis of 751 population-based studies with 4.4 million participants. Lancet 2016;387:1513-30.

2. Saaddine JB, Honeycutt AA, Narayan KM, et al. Projection of diabetic retinopathy and other major eye diseases among people with diabetes mellitus: United States, 2005-2050. Arch Ophthalmol 2008;126:1740-7.

3. Selvin E, Parrinello CM, Sacks DB, et al. Trends in prevalence and control of diabetes in the United States, 1988-1994 and 1999-2010. Ann Intern Med 2014;160:517-25.

4. Molitch ME, DeFronzo RA, Franz MJ, et al., American Diabetes Association. Nephropathy in diabetes. Diabetes Care 2004;27(Suppl 1):S79-83.

5. Klein BE. Overview of epidemiologic studies of diabetic retinopathy. Ophthalmic Epidemiol 2007;14:179-83.

6. Tesfaye S. Recent advances in the management of diabetic distal symmetrical polyneuropathy. J Diabetes Invest 2011;2:33-42.

7. Du Y, Heidemann C, Schaffrath Rosario A, et al. Changes in diabetes care indicators: findings from German National Health Interview and Examination Surveys 1997-1999 and 2008-2011. BMJ Open Diabetes Res Care 2015;3:e000135.

8. Stark Casagrande S, Fradkin JE, Saydah SH, et al. The prevalence of meeting A1C, blood pressure, and LDL goals among people with diabetes, 1988-2010. Diabetes Care 2013;36:2271-9.

9. Ali MK, Bullard KM, Saaddine JB, et al. Achievement of goals in U. S. diabetes care, 1999-2010. N Engl J Med 2013;368:1613-24.

10. Ross SA, Dzida G, Vora J, et al. Impact of weight gain on outcomes in type 2 diabetes. Curr Med Res Opin 2011;27:1431-8.

11. Kobayashi M, Yamazaki K, Hirao K, et al., Japan Diabetes Clinical Data Management Study Group. The status of diabetes control and antidiabetic drug therapy in Japan-a cross-sectional survey of 17,000 patients with diabetes mellitus (JDDM 1). Diabetes Res Clin Pract 2006;73:198-204.

12. http://www.jds.or.jp/modules/en/index.php?content_id=44 (2016/3/1 downloaded).

13. Matsuo S, Imai E, Horio M. Collaborators developing the Japanese equation for estimated GFR. Revised equations for estimated GFR from serum creatinine in Japan. Am J Kidney Dis 2009;53:982-92.

14. Grading diabetic retinopathy from stereoscopic color fundus photographs-an extension of the modified Airlie House Classification. ETDRS report number 10. Early Treatment Diabetic Retinopathy Study Research Group. Ophthalmology 1991;98(5 Suppl):786-806.

15. Yasuda H, Sanada M, Kitada K, et al. Rationale and usefulness of newly devised abbreviated diagnostic criteria and staging for diabetic polyneuropathy. Diabetes Res Clin Pract 2007;77(Suppl 1): S178-83.

16. Yokoyama H, Sone H, Oishi M, et al., Japan Diabetes Clinical Data Management Study Group. Prevalence of albuminuria and renal insufficiency and associated clinical factors in type 2 diabetes: the Japan Diabetes Clinical Data Management study (JDDM15). Nephrol Dial Transplant 2009;24:1212-19. 
17. Thomas RL, Dunstan FD, Luzio SD, et al. Prevalence of diabetic retinopathy within a national diabetic retinopathy screening service. Br J Ophthalmol 2015;99:64-8.

18. Dutra Medeiros M, Mesquita E, Papoila AL, et al. First diabetic retinopathy prevalence study in Portugal: RETINODIAB studyevaluation of the screening programme for Lisbon and Tagus Valley region. Br J Ophthalmol 2015;99:1328-33.

19. Rodriguez-Poncelas A, Miravet-Jiménez S, Casellas A, et al. Prevalence of diabetic retinopathy in individuals with type 2 diabetes who had recorded diabetic retinopathy from retinal photographs in Catalonia (Spain). Br J Ophthalmol 2015;99:1628-33.

20. Looker HC, Nyangoma SO, Cromie D, et al., Scottish Diabetic Retinopathy Screening Collaborative; Scottish Diabetes Research Network Epidemiology Group. Diabetic retinopathy at diagnosis of type 2 diabetes in Scotland. Diabetologia 2012;55:2335-42.

21. Misra A, Bachmann MO, Greenwood RH, et al. Trends in yield and effects of screening intervals during 17 years of a large UK community-based diabetic retinopathy screening programme. Diabet Med 2009;26:1040-7.

22. Bansal D, Gudala K, Muthyala $\mathrm{H}$, et al. Prevalence and risk factors of development of peripheral diabetic neuropathy in type 2 diabetes mellitus in a tertiary care setting. $J$ Diabetes Invest 2014;6:714-21.

23. Salvotelli L, Stoico V, Perrone F, et al. Prevalence of neuropathy in type 2 diabetic patients and its association with other diabetes complications: the Verona Diabetic Foot Screening Program. J Diabetes Compl 2015;29:1066-70.

24. Li L, Chen J, Wang J, et al. Prevalence and risk factors of diabetic peripheral neuropathy in type 2 diabetes mellitus patients with overweight/obese in Guangdong province, China. Prim Care Diabetes 2015;9:191-5.

25. Won JC, Kwon HS, Kim CH, et al. Prevalence and clinical characteristics of diabetic peripheral neuropathy in hospital patients with type 2 diabetes in Korea. Diabet Med 2012;29:e290-96.

26. Penno G, Solini A, Zoppini G, et al., Renal Insufficiency And Cardiovascular Events (RIACE) Study Group. Hypertriglyceridemia is independently associated with renal, but not retinal complications in subjects with type 2 diabetes: a cross-sectional analysis of the Renal Insufficiency And Cardiovascular Events (RIACE) Italian Multicenter Study. PLoS ONE 2015;10:e0125512.

27. Benarous R, Sasongko MB, Qureshi S, et al. Differential association of serum lipids with diabetic retinopathy and diabetic macular edema. Invest Ophthalmol Vis Sci 2011;52:7464-9.

28. Wu M, Chen $\mathrm{Y}$, Wilson $\mathrm{K}$, et al. Intraretinal leakage and oxidation of LDL in diabetic retinopathy. Invest Ophthalmol Vis Sci 2008;49:2679-85.
29. Haslam DW, James WP. Obesity. Lancet 2005;366:1197-209.

30. Ezzati M, Lopez AD, Rodgers A, et al., Comparative Risk Assessment Collaborating Group. Selected major risk factors and global and regional burden of disease. Lancet 2002;360:1347-60.

31. Ferrannini E, Cushman WC. Diabetes and hypertension: the bad companions. Lancet 2012;380:601-10.

32. Rüster $\mathrm{C}$, Wolf $\mathrm{G}$. The role of the renin-angiotensin-aldosterone system in obesity-related renal diseases. Semin Nephrol 2013;33:44-53.

33. Choi JA, Ko SH, Park YR, et al. Retinal nerve fiber layer loss is associated with urinary albumin excretion in patients with type 2 diabetes. Ophthalmology 2015;122:976-81.

34. Rodríguez-Poncelas A, Mundet-Tudurí X, Miravet-Jiménez S, et al. Chronic kidney disease and diabetic retinopathy in patients with type 2 diabetes. PLOS ONE 2016;11:e0149448.

35. Mottl AK, Kwon KS, Garg S, et al. The association of retinopathy and low GFR in type 2 diabetes. Diabetes Res Clin Pract 2012;98:487-93.

36. Gimeno-Orna JA, Faure-Nogueras E, Castro-Alonso FJ, et al. Ability of retinopathy to predict cardiovascular disease in patients with type 2 diabetes mellitus. Am J Cardiol 2009;103:1364-7.

37. Drury PL, Ting R, Zannino D, et al. Estimated glomerular filtration rate and albuminuria are independent predictors of cardiovascular events and death in type 2 diabetes mellitus: the Fenofibrate Intervention and Event Lowering in Diabetes (FIELD) study. Diabetologia 2011;54:32-43.

38. Yokoyama H, Araki S, Haneda M, et al., Japan Diabetes Clinical Data Management Study Group. Chronic kidney disease categories and renal-cardiovascular outcomes in type 2 diabetes without prevalent cardiovascular disease: a prospective cohort study (JDDM25). Diabetologia 2012;55:1911-18.

39. Jaiswal M, Fufaa GD, Martin CL, et al. Burden of diabetic peripheral neuropathy in Pima Indians with type 2 diabetes. Diabetes Care 2016;39:e63-4

40. Brownrigg JR, Hughes $\mathrm{CO}$, Burleigh D, et al. Microvascular disease and risk of cardiovascular events among individuals with type 2 diabetes: a population-level cohort study. Lancet Diabetes Endocrinol 2016;4:588-97.

41. Kaiser N, Sasson S, Feener EP, et al. Differential regulation of glucose transport and transporters by glucose in vascular endothelial and smooth muscle cells. Diabetes 1993;42:80-9.

42. Hammes HP, Federoff HJ, Brownlee M. Nerve growth factor prevents both neuroretinal programmed cell death and capillary pathology in experimental diabetes. Mol Med 1995;1:527-34.

43. Ceriello $A$. The possible role of postprandial hyperglycaemia in the pathogenesis of diabetic complications. Diabetologia 2003;46 (Suppl 1):M9-16. 